\title{
A neutron monitor as an integral spectrometer for GLE analysis: Effective rigidity and reassessment of integral fluxes
}

\author{
Sergey A. Koldobskiy* \\ Oulu University, Oulu, Finland and National Research Nuclear University MEPhI, Moscow, \\ Russia \\ E-mail: sakoldobskiy@mephi.ru
}

\author{
Alexandar Mishev \\ Oulu University, Oulu, Finland
}

\section{Gennady A. Kovaltsov}

Ioffe Physical-Technical Institute, St. Petersburg, Russia

Ilya G. Usoskin

Oulu University, Oulu, Finland

\begin{abstract}
In this work a new concept of the effective rigidity, $R_{\text {eff }}$, of a neutron monitor (NM) for detection of a ground-level enhancement (GLE) is introduced so that the integral omnidirectional flux of solar energetic particles (SEP) with rigidity above it is proportional to the NM response to GLE. The effective rigidity depends on the location (the cutoff rigidity and altitude) of the NM but is independent on the SEP spectrum in a broad range of the spectral parameters. Use of this concept allows one to directly evaluate the SEP fluence. The exact value of $R_{\text {eff }}$ depends on the NM yield function. We have tested four modern yield function: Mi13 (Mishev et al., JGR:SP, 2013), Ma16 (Mangeard et al., JGR:SP, 2016), CM12 (Caballero-Lopez \& Moraal, JGR:SP, 2012) and CD00 (Clem \& Dorman, SSR, 2000) and recommend Mi13 and CM12 for practical use. The method was applied to three recent GLEs: \# 69, 70 and 71, for which a reconstruction of the energy spectra without any assumptions on the exact spectral shape was performed. We found that the SEP fluence is not well described by a power-law function but demonstrates a steepening at higher energies, which can be approximated by the modified power-law or Ellison-Ramaty functions.
\end{abstract}

36th International Cosmic Ray Conference -ICRC2019-

July 24th - August 1st, 2019

Madison, WI, U.S.A.

${ }^{*}$ Speaker. 


\section{Introduction}

Fluxes of the galactic cosmic rays (GCR) are permanently bombard Earth with the intensity modulated by solar activity in the course of the 11-year solar cycle. If these particles have sufficient energy they can initiate the cascade generating the secondary nucleonic cosmic-ray component. These secondary particles can be registered by the neutron monitors, which are united into the worldwide network and are used for continuous monitoring of cosmic-ray fluxes.

Sometimes sporadic fluxes of solar energetic particles (SEPs) caused by extreme solar events like solar flares or coronal mass ejections can also reach Earth. During these SEP events, fluxes of low energy particles (below several hundred $\mathrm{MeV}$ ) can enhance over the GCR background by many orders of magnitude during several to tens of hours. For the SEP events the NM response can be registered only for hard-spectrum SEP events, which can initiate the atmospheric cascade and be detected by ground-based NMs, these events is called Ground-Level Enhancements or GLEs. At present there are known 72 such events and the data for 67 events is available (a list can be found at https://gle.oulu.fi).

The SEP events study is important for different reasons, from academic, such as studying solar eruptive events and probing the inner heliosphere, to practical ones, since these fluxes due to their high intensities produce serious radiation hazards for space-based technologies.

SEP events are continuously monitored by space missions over the last several decades. Due to mass/cost limitations most space missions are able to measure mainly the low-energy, $\leq 100 \mathrm{MeV}$, range of particles. Two recent missions are/were able to measure energetic cosmic-ray particles in space: PAMELA was in operation from June 2006 through January 2016, while AMS-02 is in operation since 2011. But both missions are not well suited for SEP monitoring because of their low orbits, whose major fraction is located inside the geomagnetic field and is thus protected from low-energy cosmic particles by the Earth's magnetosphere.

So the only one type of detectors able to continuously monitor the energy range above several hundred $\mathrm{MeV}$ is a ground-based neutron monitor (NM). On one hand, NM is an energy-integrating detector unable to directly measure the particle energy/rigididty spectrum. On the other hand, the world-wide network of NMs, located in different places with different geomagnetic rigidity cutoffs, allows one to roughly assess the spectrum of charged particles during SEP events. The key here is the knowledge of the yield function (YF) of a NM that quantifies the response of a NM to a monoenergetic unit flux of primary energetic particles on the top of the atmosphere.

Knowing the NM YF, the neutron monitor count rate can be computed as:

$$
N\left(P_{\mathrm{c}}, h\right)=\sum_{j} \int_{P_{\mathrm{c}}}^{\infty} J_{j}(R) \cdot Y_{j}(R, h) \cdot d R
$$

where $Y_{j}(R, h)$ is the yield function of the NM (located at height $h$ ) for primary cosmic-ray particles of type $j$ (protons, helium, heavier species), and $J_{j}$ is the differential rigidity intensity of primary particles of type $j$ at the Earth's orbit but outside the magnetosphere and atmosphere. Both GCR and SEP NM responses can be calculated using YFs.

Usually, the spectrum of SEPs is reconstructed parameterically, in this case spectral shape of SEP is prescribed and best-fitting procedures are used to find the fit parameters. Usually in this approach the SEP pitch-angle anisotropy is considered, which can be large in the initial impulsive 
phase of the event [1]. This method, while allowing for estimate of the time-variable spectral and angular distributions of SEPs during the events, is laborious and not always stable, that may lead to large uncertainties, mostly due to differences in NM yield functions.

For practical applications, it is often sufficient to know only the flux integrated over the entire event (it is called event fluence). Determination of the event fluence is more robust in this case and is usually done under an assumption of the isotropic distribution of SEP particles near Earth. A detailed method for this kind of analysis was proposed by Tylka and Dietrich [2]. They used a power-law function as a prescribed SEP fluence function to describe the high-energy tail where the NM responses can be used. Results of this work were summarized in [3] (hereafter R18), where they are combined with the satellite measurements data and the Band function was used to unite NM and satellite datasets. Despite the high importance of this work, there are several issues: first, this method is parametric (it is based on an assumption of the power-law spectral shape) and, second, it uses an outdated yield function of Clem \& Dorman [4].

Here we propose a further development of the R18/Tylka and Dietrich method [2, 3], by introducing the effective rigidity of a $\mathrm{NM}$, that enables one to make a non-parametric reconstructions of the GLE integral fluence, based on the data from the NM network, and use the modern yield function, validated on the satellite data.

In this report only a short description of obtained results is presented, for a more detailed description of methods and results please refer to [5, 6].

\section{Effective rigidity method}

The new approach makes it possible to assess the SEP energy-integrated spectrum directly, without making any assumption of the spectral shape. Let us assume that there is an effective rigidity $R_{\text {eff }}$ such that the integral fluence of SEPs above this rigidity $F\left(>R_{\text {eff }}\right)$, is proportional to the NM response to GLE $N_{\mathrm{GLE}}$ :

$$
F\left(>R_{\mathrm{eff}}\right)=K_{\mathrm{eff}} \cdot N_{\mathrm{GLE}},
$$

where $K_{\text {eff }}$ is a scaling factor for a given NM, which is ideally a constant irrespectively of the strength and energy spectrum of the analyzed event [5]. The expected response of a NM to GLE is calculated using Equation 1.1. We assume that SEPs causing the GLE consists of protons only.

We consider the modified power law in rigidity as the spectral shape of SEP fluence:

$$
\frac{d F}{d R}=F_{0} R^{-(\gamma+\delta \gamma(R-1))}
$$

where $R$ is rigidity in GV, $\gamma$ is the spectral index, and $\delta \gamma$ in $\mathrm{GV}^{-1}$ is the rate of the spectrum steepening. In the forthcoming analysis we varied the value of $\gamma$ in a range from 5 to 9 , which corresponds to typical GLE spectra. The rate of the spectrum steepening $\delta \gamma$ was considered in the range from 0 to $1 \mathrm{GV}^{-1}$. The analyzed range of $\gamma$ and $\delta \gamma$ corresponds to a wide range spectra of real SEP events. For each value of $\gamma$ and $\delta \gamma$ from these ranges we calculated the value of $K$ for different values of $R$

$$
K(R, \gamma, \delta \gamma)=\frac{F(>R)}{N_{\mathrm{GLE}}},
$$


where $N_{\mathrm{GLE}}$ is defined using Equation 1.1. This forms a ribbon in the $K-\mathrm{vs}-R$ diagram (see Figure 1). We considered the vertical full-range width of the ribbon, for a given $R$, as $\Delta K$. Next we found such a value of $R$, called the effective rigidity $R_{\text {eff }}$, which minimizes the value of $\delta K(R) \equiv \Delta K /\langle K\rangle$, where $\langle K\rangle$ is a mean value of $K$ for the given value of $R$. The value of $\langle K\rangle$ is called the effective scaling factor $K_{\text {eff }}$. Full-scale uncertainties for both values $R_{\text {eff }}$ and $K_{\text {eff }}$ were evaluated as illustrated by the red error bars in Figure 1.
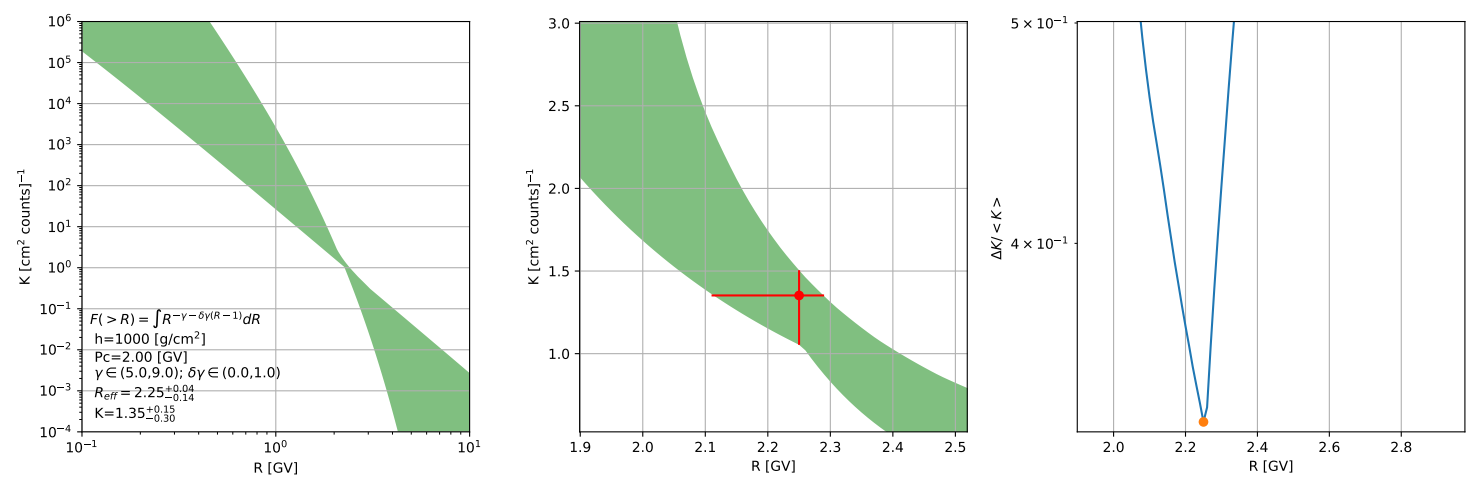

Figure 1: Left: diagram of the scaling factor $K$ versus rigidity $R$ for the standard near-sea-level 1NM64 $\left(P_{\mathrm{c}}=2 \mathrm{GV}, \mathrm{h}=1000 \mathrm{~g} / \mathrm{cm}^{2}\right)$, using the Mi13 YF, for the spectral indices (equation 2.2) $\gamma$ and $\delta \gamma$ ranging between 5-9 and $0-1$, respectively. $R_{\text {eff }}$ and the corresponding value of $K_{\mathrm{eff}}$, with full-range uncertainties is denoted by the red dot. Middle: zoom to the left-hand panel around the $R_{\text {eff }}$ value. Left: $\Delta K /\langle K\rangle$ as a function of rigidity.

In a similar way we have defined effective rigidities and scaling factors for different geomagnetic rigidity cutoffs $P_{\mathrm{c}}$ and altitudes $h$, as shown in Figure 2 for Mi13 YF. Since the Mi13 yield function was computed only for the sea-level NMs, we used the altitudinal dependence according to Flückiger et al. [7] applied to the Mi13 yield function. One can see that the effective rigidity $R_{\text {eff }}$ is very close to the geomagnetic rigidity cutoff $P_{\mathrm{c}}$ for low- and mid-latitude locations $\left(P_{\mathrm{c}}>3\right.$ $\mathrm{GV}$ ) but saturates at 1.3-1.5 GV (depending on the atmospheric depth) for high-latitude sites. The value of the $K_{\text {eff }}$ varies with the geomagnetic cutoff depicting a shoulder at high-latitude locations and a nearly exponential decrease with $P_{\mathrm{c}}$ for low- and mid-latitudes. This relation is shaped by two different processes, viz. the atmospheric cutoff and the geomagnetic cutoff. While the geomagnetic cutoff dominates at low- and mid-latitudes, the atmospheric cutoff becomes crucial at high latitudes.

It is important that the effective rigidity and scaling factor are defined robustly for a wide range of the geomagnetic rigidity cutoffs, and they are independent on the exact SEP spectrum, in a reasonable range of parameters. Thus, for each GLE and each NM, one can estimate, using Equation 2.1, the integral fluence $F\left(>R_{\text {eff }}\right.$ ) of SEPs, and a set of such NMs with different values of $R_{\text {eff }}$ makes it possible to perform a non-parametric reconstruction of the event's integral spectrum.

\section{Reconstruction of the GLE integral fluxes}

To reconstruct the GLE integral flux, we need to know the relative measured response of $i$-th 

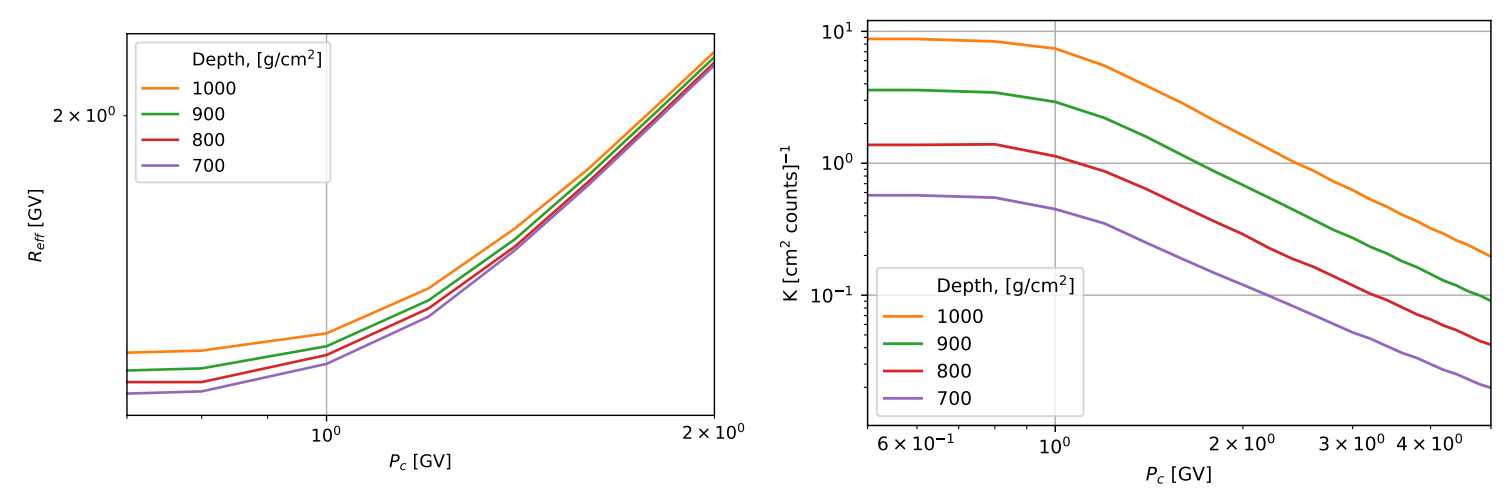

Figure 2: Left: the effective rigidity $R_{\text {eff }}$ of a NM, using the Mi13 YF, as a function of the cutoff rigidity $P_{\mathrm{c}}$ for different atmospheric depths as denoted in the legend. Right: the same for the $K_{\text {eff }}$.

NM to a GLE, integrated over the entire event. It is defined as

$$
\left(\frac{N_{\mathrm{GLE}, i}}{N_{\mathrm{GCR}, i}}\right)_{\exp }=\frac{3600 \cdot X_{i}}{100},
$$

where $X_{i}$ is the event-integrated relative intensity of GLE in units of percent-hours, where percents are with respect to the pre-increase NM count rate due to GCR. $X_{i}$ and corresponding integrated GLE responses are measured experimentally. We note that these values were reconstructed independently from those used in R18 and may differ. Geomagnetic cutoff rigidities $P_{\mathrm{c}}$ were taken from NMDB database.

The theoretical NM response to GLE $N_{\mathrm{GLE}}$, which enters Equation 2.1, is

$$
N_{\mathrm{GLE}}=N_{\mathrm{GCR}} \cdot\left(\frac{N_{\mathrm{GLE}}}{N_{\mathrm{GCR}}}\right)_{\exp },
$$

where $N_{\mathrm{GCR}}$ was calculated using Eq. 1.1 with modern local interstellar spectrum by Vos $\&$ Potgieter [8] in solar modulation potential $\phi$ approach. $\phi$ values were adopted from [9].

Statistical uncertainties of $N_{\mathrm{GLE}}$ have two independent sources: the accuracy of the determination of $X_{i}$ considered as $1 \% \mathrm{hr}$ and the statistical uncertainty of the GCR count rate:

$$
\sigma_{N_{\mathrm{GLE}, i}}=\sqrt{\left(0.01 N_{\mathrm{GCR}, i}\right)^{2}+N_{\mathrm{GCR}, i}} .
$$

Another source of uncertainties of the final reconstruction are the uncertainties in definition of $R_{\text {eff }}$ and $K_{\text {eff }}$ and all the uncertainties were accounted for in the following analysis.

We have tested the $R_{\text {eff }}$ method for three well-studied events: GLE \#69, 70 and 71 using four modern yield functions: Mi13 [10], Ma16 [11], CM12 [12], CD00 [4]. In Figure 3 we show the reconstructed integral fluence for GLE \#71. The ratios to R18 for fluence reconstructed with the $R_{\text {eff }}$ method using different YF denoted with colored points along with 1- $\sigma$ fit of direct PAMELA measurements [13] (grey area) and full reconstruction using Mi13 YF [1] (dotted red line) are shown. One can see that the spectra, reconstructed using the $R_{\text {eff }}$ method, are fully consistent with the direct data (grey area) in the spectral shape, but those based on CD00 and Ma16 YFs tend to underestimate the low-energy range of the spectrum. On the other hand the Band-function 
approximation (R18) systematically underestimates, by the factor $2-3$, the fluence in the rigidity range of about $2 \mathrm{GV}$ (energy about $1 \mathrm{GeV}$ ) and lies very close to the values, based on $R_{\text {eff }}$ method using the Mi16 and CD00 YFs. The full NM-based reconstruction based on Mi13 YF (dashed red line) demonstrates the excellent agreement with the spectrum reconstructed here, and a fair agreement, within the uncertainties, with the directly observed one.

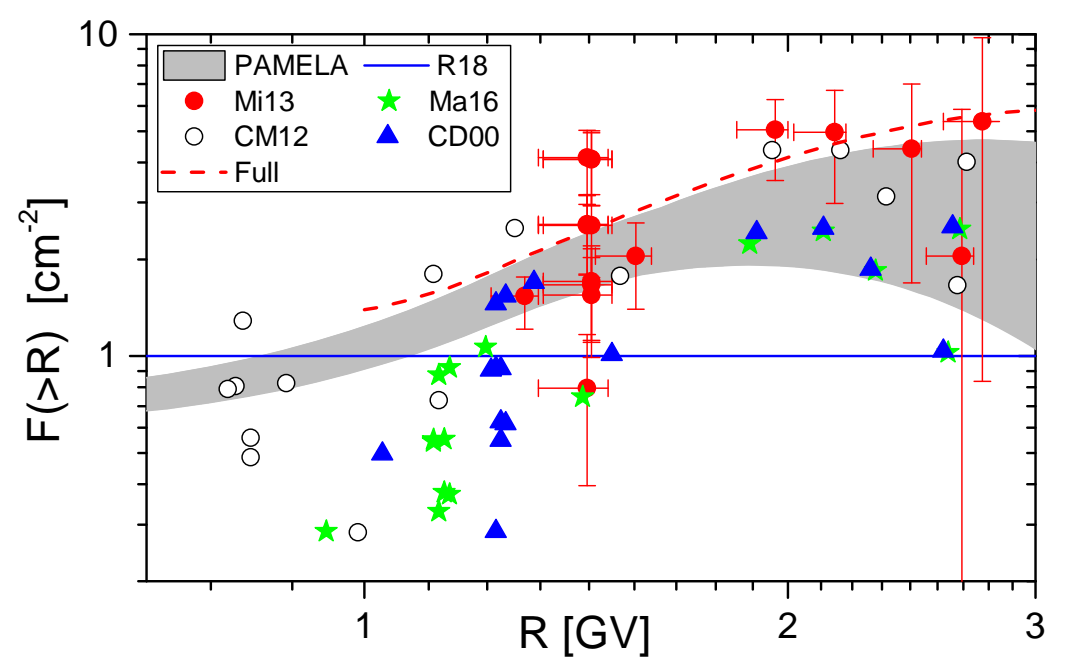

Figure 3: Reconstruction of the integral SEP fluence for GLE \#71 (17-May-2012), divided by the R18 reconstruction. Colored points denotes the reconstruction using the effective-rigidity $R_{\text {eff }}$ method using different yield functions (errors bars are shown only for the Mi13 YF, for clarity), along with full reconstruction based on the Mi13 YF (red dashed line). Direct measurements by the PAMELA experiment parameterized via the Ellison-Ramaty form with $1 \sigma$ uncertainties are shown with the grey-filled area [13].

In Figure 4 the fluence reconstructed with $R_{\text {eff }}$ method is shown, divided by the Band function from R18, for GLE \#69 and \#70 and the difference by the factor 2-4 is clearly visible. Moreover, for these two GLEs the significant difference from the single power law (that corresponds to the tail of Band function) arises.

\section{Discussion and conclusion}

In this report we have presented a new fast method for assessment of the high-rigidity part (above $1 \mathrm{GV}$ ) of the spectral fluence of SEP for GLE events using the data from the worldwide NM network. The method is based on the effective rigidity $R_{\text {eff }}$ and scaling factor $K_{\text {eff }}$, which are calculated for each NM independently, so the SEP integral fluence is directly proportional to the NM response to the event. This method is non-parametric so that it provides an estimate of the spectrum without any assumption of the spectral shape. This method is simple and fast in calculations avoiding laborious computations needed for the full reconstruction, but it neglects the possible anisotropy during the event.

We have made reconstruction of the SEP fluence for GLE \#69 (20-Jan-2005), 70 (13-Dec2006) and 71 (17-May-2012), using the $R_{\text {eff }}$ method. For GLE \#71 we have compared obtained 

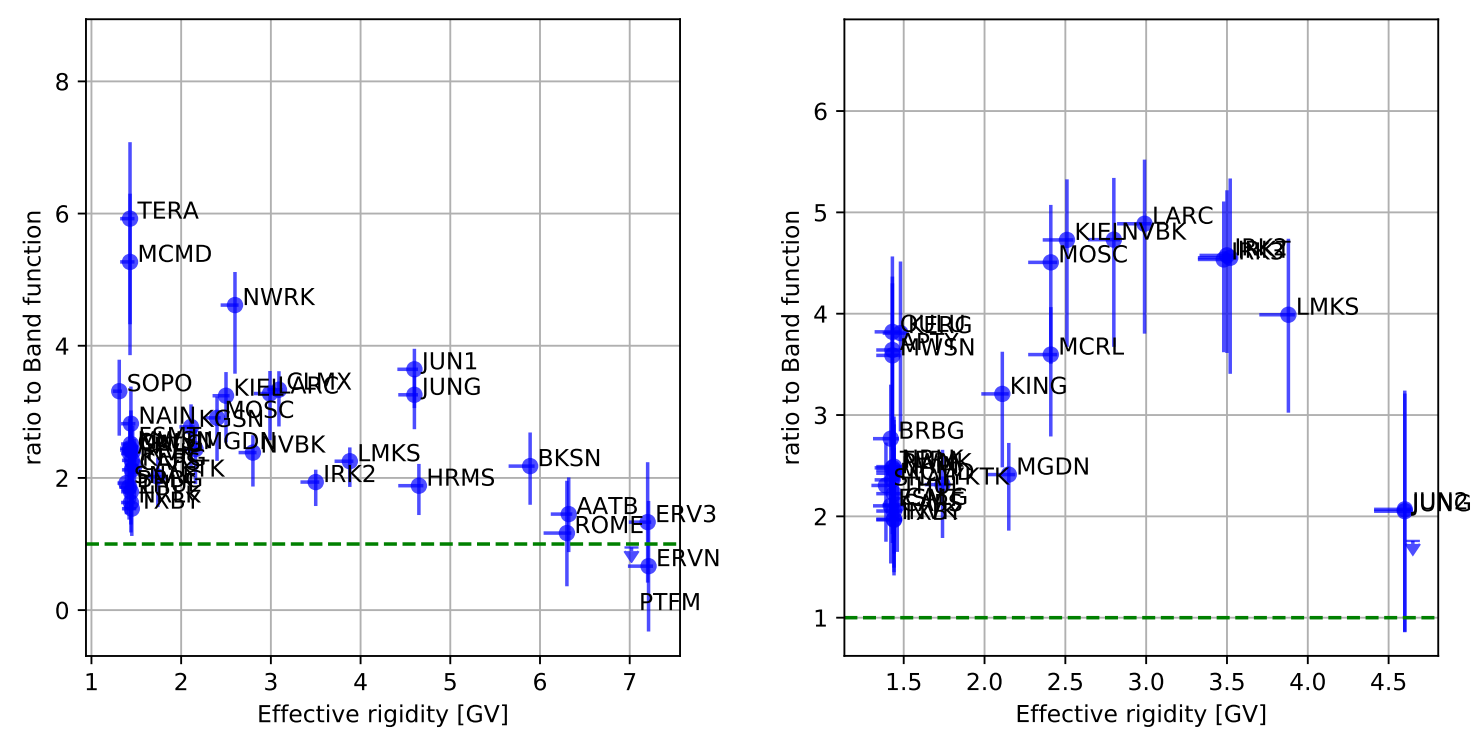

Figure 4: Reconstruction of integral SEP fluences by the effective-rigidity $R_{\text {eff }}$ method, divided by the R18 reconstruction. Left: for the GLE \#69 (20-Jan-2005). Right: for GLE \#70 (13-Dec-2006).

result with those directly measured by PAMELA experiment. Four modern YFs were tested for this comparison. Only the reconstructions based on Mi13 and CM12 YFs appear quantitatively consistent with the PAMELA measurements, while the results based on CD00 and Ma16 lead to an underestimate of the spectrum by a factor $2-3$ in the lower rigidity range. The systematic difference between spectral shapes for GLE \#71, obtained, on one hand, in this work, using the direct reconstruction and in PAMELA experiment, and, on another hand, in R18, is probably related to the fact that the latter is based on the CD00 YF. CD00 and Mi13 YFs tend to overestimate the NM response to lower energy particles, as was found by Koldobskiy et al. [14] from an analysis of GCR spectra measured directly by AMS-02 and PAMELA.

Reconstruction for GLE \#69 and \#70 showed that the power-law approximation of the highenergy tail, used in particular in the Band-function parameterization, does not properly describe the form of the GLE spectrum in the NM-energy range, so the earlier estimates of GLE integral fluences need to be revised. A proper reconstruction of the SEP integral fluence for the known GLE events is planned for a forthcoming work.

\section{Acknowledgments}

Data of GLE recorded by NMs were obtained from the International GLE database http: //gle.oulu.fi and NMDB database (www.nmdb.eu, founded under the European Union's FP7 programme, contract no. 213007). PIs and teams of all the ground-based neutron monitors whose data were used here, are gratefully acknowledged. This work was partially supported by the ReSoLVE Centre of Excellence (project no. 307411) and HEAIM Project (no. 314982 and no. 316223) of Academy of Finland, by the grant no. MK-6160.2018.2 of the President of the Russian Federation and MEPhI Academic Excellence Project (contract 02.a03.21.0005). 


\section{References}

[1] Alexander Mishev, Sasu Tuohino, and Ilya Usoskin. "Neutron monitor count rate increase as a proxy for dose rate assessment at aviation altitudes during GLEs". In: Journal of Space Weather and Space Climate 8 (2018), A46.

[2] A. Tylka and W. Dietrich. "A new and comprehensive analysis of proton spectra in groundlevel enhanced (GLE) solar particle events". In: Proceedings of the 31st ICRC, Lodz (2009), pp. $1-4$.

[3] Osku Raukunen et al. "Two solar proton fluence models based on ground level enhancement observations”. In: Journal of Space Weather and Space Climate 8 (2018), A04.

[4] John M. Clem and Lev I. Dorman. "Neutron monitor response functions". In: Space Science Reviews 93.1-2 (2000), pp. 335-359.

[5] Sergey A. Koldobskiy, Gennady A. Kovaltsov, and Ilya G. Usoskin. "Effective Rigidity of a Polar Neutron Monitor for Recording Ground-Level Enhancements". In: Solar Physics 293.7 (2018), p. 110.

[6] Sergey A. Koldobskiy et al. "New Method of Assessment of the Integral Fluence of Solar Energetic (> 1 GV Rigidity) Particles from Neutron Monitor Data”. In: Solar Physics 294.7 (2019), p. 94.

[7] E O Fluckiger et al. "A parameterized neutron monitor yield function for space weather applications". In: Proceedings of the 30th International Cosmic Ray Conference. Ed. by Gustavo Medina-Tanco Rogelio Caballero, Juan Carlos D’Olivo and José F. Valdés-Galicia Lukas Nellen, Federico A. Sánchez. Mexico City, 2008, pp. 289-282.

[8] Etienne E. Vos and Marius S. Potgieter. "NEW MODELING OF GALACTIC PROTON MODULATION DURING THE MINIMUM OF SOLAR CYCLE 23/24”. In: The Astrophysical Journal 815.2 (2015), p. 119.

[9] Ilya G Usoskin et al. "Heliospheric modulation of cosmic rays during the neutron monitor era: Calibration using PAMELA data for 2006-2010”. In: Journal of Geophysical Research: Space Physics 122.4 (2017), pp. 3875-3887.

[10] A L Mishev, I G Usoskin, and G A Kovaltsov. "Neutron monitor yield function: New improved computations". In: Journal of Geophysical Research: Space Physics 118.6 (2013), pp. 2783-2788.

[11] P.-S. Mangeard et al. "Monte Carlo simulation of the neutron monitor yield function". In: Journal of Geophysical Research: Space Physics 121.8 (2016), pp. 7435-7448.

[12] R. A. Caballero-Lopez and H. Moraal. "Cosmic-ray yield and response functions in the atmosphere”. In: Journal of Geophysical Research: Space Physics 117 (2012), A12103.

[13] A. Bruno et al. "Solar Energetic Particle Events Observed by the PAMELA Mission". In: The Astrophysical Journal 862.2 (2018), p. 97. arXiv: 1807.10183.

[14] Sergey A. Koldobskiy et al. "Validation of the Neutron Monitor Yield Function Using Data From AMS-02 Experiment, 2011-2017”. In: Journal of Geophysical Research: Space Physics 124.4 (2019), pp. 2367-2379. 